Thorax (1966), 21, 511.

\title{
Diffuse oesophageal spasm
}

\author{
D. R. CRAD DOCK, A. LOGAN, AND P. R. WALBAUM
}

From the Thoracic Unit, Royal Infirmary, Edinburgh

Incoordination of muscular contraction is sometimes seen in the apparently healthy oesophagus, but it is a prominent feature of two conditionsachalasia and diffuse spasm.

This review is concerned with the second of these. In the 12-year period 1954-66, 12 cases of diffuse spasm were recorded in our unit. Le Roux and Wright (1961) reported on 55 cases of achalasia in the same unit during the 10-year period 1948-58. In our experience, achalasia, which is a rare disease, is approximately five times more common than diffuse oesophageal spasm. This is the incidence of the two conditions as seen in a thoracic surgical unit, but the radiological pattern of 'corkscrew oesophagus' is seen frequently in the course of barium examination in patients without dysphagia (Sheinmel, Priviteri, and Poppel, 1949). The patients referred to in this report all presented with dysphagia and demonstrated the radiological pattern and intraluminal oesophageal pressure tracings of diffuse spasm.

\section{CLINICAL MATERIAL}

Twelve patients have been reviewed. They were investigated and managed with a uniform policy. All had a barium swallow examination and oesophagoscopy. All except one had oesophageal pressure tracings recorded. Unfortunately, two of the earlier patients subjected to operation did not have oesophageal pressure measurements preoperatively.

\section{CLINICAL FEATURES}

AGE AND SEX Ages ranged from 44 to 69 years at the time of initial presentation. There were six women and six men.

FAMILY HISTORY Only one patient, a 62-year-old woman, gave a family history of oesophageal disease. Her mother had died of carcinoma of the oesophagus.
SYMPTOMS Dysphagia was the symptom common to all these patients. It was intermittent and varied in severity. It was rarely severe enough to have an appreciable effect on nutrition, but two patients had lost over 2 stones $(12.7 \mathrm{~kg}$.) in weight before presentation. Most claimed to have come to terms with their dysphagia and, although it prevented their enjoyment of normal meals, they adjusted their diet accordingly. Two had the unusual complaint of difficulty in swallowing liquids, whereas solids were managed relatively well. The commonest site of food 'sticking' was behind the xiphisternum. The interval between episodes of dysphagia varied, some claiming difficulty at every meal and some swallowing normally for three to four days, with all ranges in between. During exacerbations, or else when the patients became weary of their symptoms, they presented for hospital treatment.

The duration of dysphagia varied from eight weeks to 15 years, with an average of four years, before the first examination. All patients had some regurgitation, and in three there was also a considerable amount of acid reflux and heartburn. Pain on swallowing was not always a feature, and in this respect our series differs from that of Creamer, Donoghue, and Code (1958), who reported that 13 of their 16 patients complained of pain. It is difficult to separate pain from discomfort during swallowing, but in our series only four patients admitted painful swallowing.

\section{INVESTIGATIONS}

BARIUM SWALLOW The characteristic appearance produced by diffuse oesophageal spasm during a barium swallow is one of incoordinated, irregular contractions of the oesophagus most marked from the aortic arch to the diaphragm. The consequent segmentation of barium gives rise to the name 'corkscrew oesophagus'. A second but less common appearance is of tight contraction of the oesophagus over a length of several centimetres. 
This incoordinate action may cause the swallowed barium to be squeezed back into the upper oesophagus and is sometimes eliminated by a normal peristaltic wave (Figs 1 to 3 ).

Simultaneous and repetitive contractions may continue for a considerable period after a single swallow.

Although Ellis, Olsen, Holman, and Code (1958) stated that dilatation is rare in this condition, four out of the 12 patients in this series developed it in varying degrees (Figs 4 and 5).

As the disease progresses diverticula may form, and in three of the 12 patients this occurred, in two in a gross degree (Figs 1 and 6).

Johnstone (1950) and Creamer (1962) pointed out the association of diffuse spasm with hiatal hernia, and in two of the patients there was an associated sliding hiatal hernia. In a third, the picture of diffuse spasm developed only after repair of a para-oesophageal hiatal hernia.
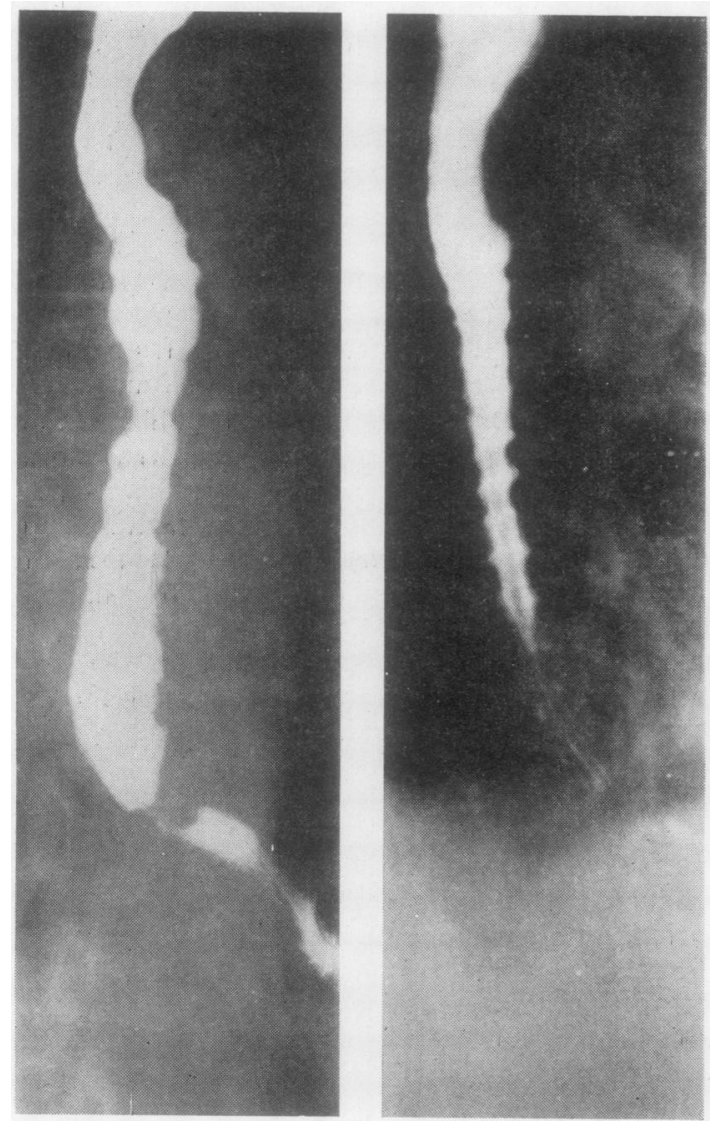

FIG. 1
One patient in this series was initially regarded, on radiological evidence, as suffering from $\frac{\bar{\omega}}{5}$ achalasia, but subsequently developed the charac- $\mathbb{D}_{0}$ teristic radiological and manometric features of diffuse spasm.

OESOPHAGOSCOPY The findings at oesophago- $-\frac{\vec{\omega}}{\omega}$ scopy were often surprisingly normal. In the twoo patients with a sliding hiatal hernia the diagnosis $\vec{x}$ was confirmed by finding a high mucosal transi-i tion. In the three patients with oesophageal dilatation there was food residue and excess salivacr in the upper oesophagus. In three, narrowed $\overrightarrow{-}$ segments were encountered in the lower half of 0 the oesophagus, but in only one was it impossibleto pass the endoscope into the stomach; this was $Z$ in the patient whose radiographs are shown in? Fig. 6 and who had the most severe form of the $\frac{\mathbb{D}}{3}$ disease. In the others the endoscopic findings were normal.
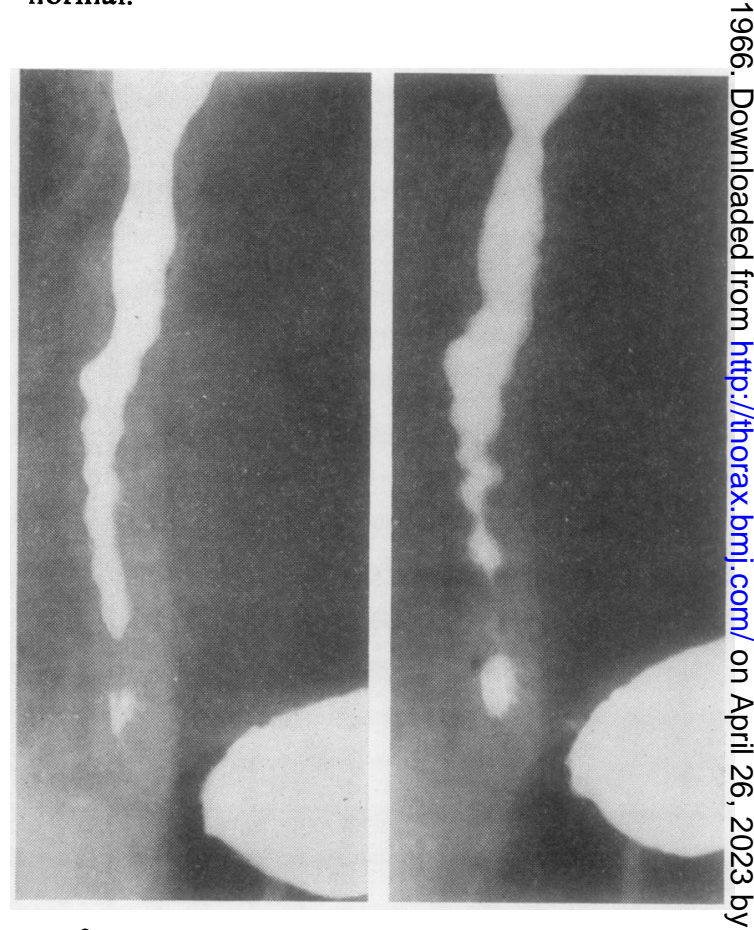

FIG. 2

FIGS 1 AND 2. Barium swallow radiographs of two $\frac{\overline{0}}{\bar{Q}}$ patients with diffuse oesophageal spasm illustrating the $\frac{\rho}{1}$ multiple, irregular, segmental oesophageal contractions $\propto$ that are a feature of the disease. It is from this appearance that the name 'corkscrew oesophagus' originates. 

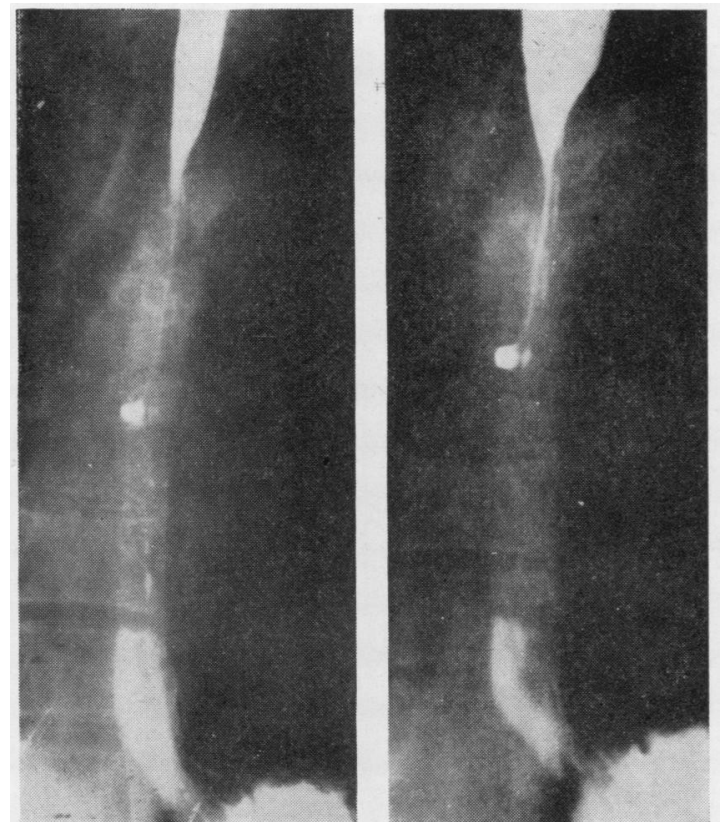

FIG. 3. Barium swallow radiograph of a patient with diffuse oesophageal spasm showing the appearances less commonly found, namely a long, spastic oesophageal segment which is in this case associated with a small diverticulum.

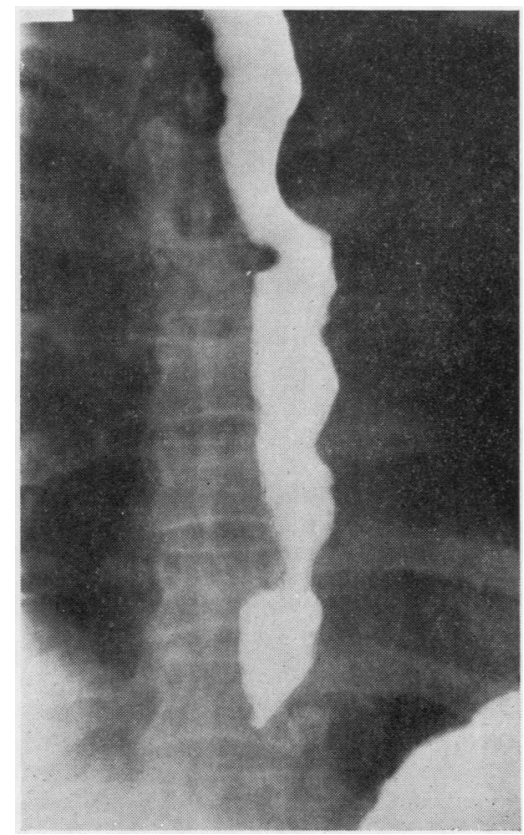

FIG. 4. Early oesophageal dilatation occurring in a patient with diffuse oesophageal incoordination.

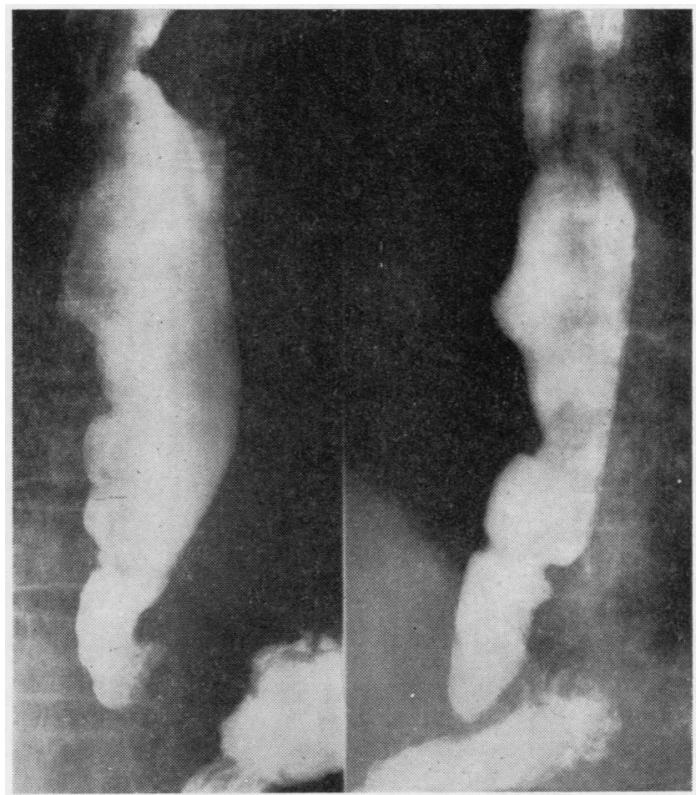

FIG. 5. Advanced oesophageal dilatation occurring in a patient with diffuse oesophageal incoordination. These radiographs are of the same patient as the radiograph in Fig. 4 but at a later stage.

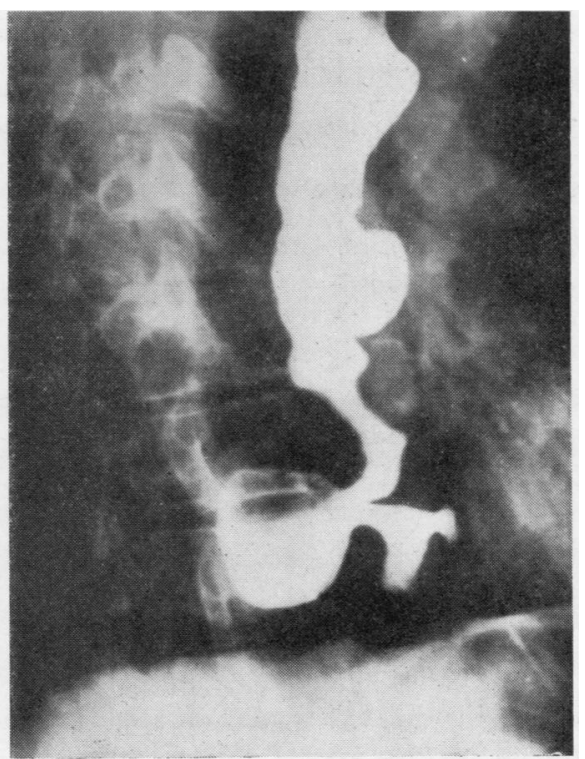

FIG. 6. Barium swallow radiograph shows the gross oesophageal deformity which may ultimately occur in a severe case of diffuse oesophageal spasm. The large oesophageal diverticulum was excised at the time of lower oesophagomyotomy. 
OESOPHAGEAL PRESSURE MEASUREMENTS These were made in 11 patients; in the twelfth it was not possible to pass the tube. The pressures were recorded by means of three balloons spaced at $5-\mathrm{cm}$. intervals on a triple-lumen tube connected to pressure transducers. The tracings obtained all showed the same features. The normal pattern of peristaltic contractions produced by swallowing (Fig. 7) was replaced by simultaneous contrac-

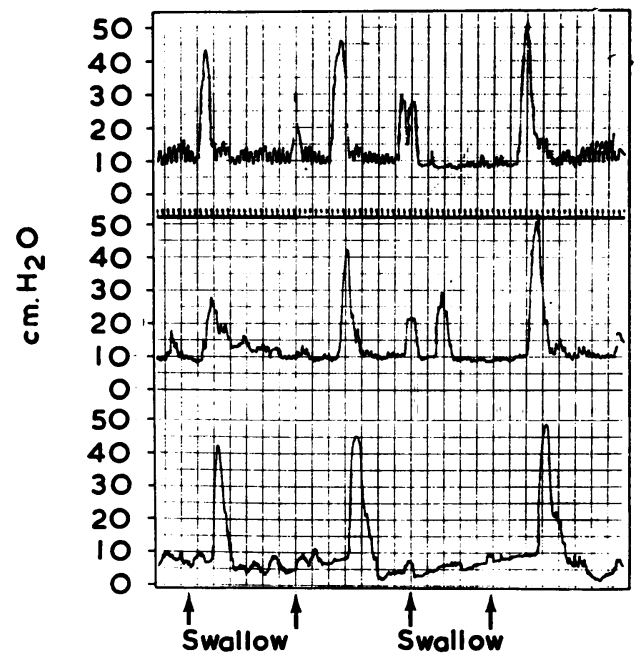

FIG. 7. Oesophageal pressure tracings showing normal progressive peristaltic contractions in a normal person. The aortic arch is approximately $30 \mathrm{~cm}$. from the nose and the cardia approximately $45 \mathrm{~cm}$. from the nose. The important features are the normal pressures, the sequential appearance of the peristaltic peaks as the wave advances down the oesophagus, and the fact that one swallow evokes only one set of contractions. Upper tracing, 30 $\mathrm{cm}$. from nose; middle tracing, $35 \mathrm{~cm}$. from nose; lower tracing, $40 \mathrm{~cm}$. from nose.

tions, often of increased amplitude. In several cases peak pressures of 150 to $200 \mathrm{~cm} . \mathrm{H}_{2} \mathrm{O}$ were obtained, although in the majority pressures were at the upper limit of normal. In addition, a single swallow often produced a series of repetitive contractions which persisted for varying periods (Figs 8 and 9). These changes were always most marked in the lower half of the oesophagus, and in some cases normal peristaltic contractions occurred in the upper half. The pattern in patients with a demonstrable hiatal hernia differed in no way from that in patients without a hernia.

\section{TREATMENT}

Two methods of treatment were used-oesophageal dilatation and lower oesophagomyotomy.

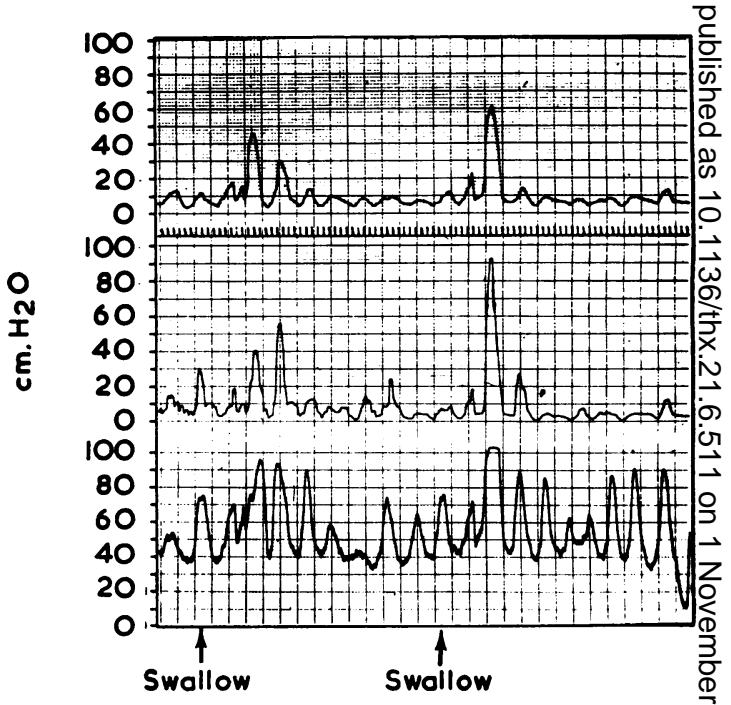

FIG. 8. Abnormal pressure tracings from a patient with diffuse oesophageal spasm. The lower tracing shows the characteristic feature of the disease; non-progressive contractions, high pressures, and the multiple contractions that follow a single swallow. Upper tracing, $30 \mathrm{~cm}$ from nose; middle tracing, $35 \mathrm{~cm}$. from nose; lowe tracing, $40 \mathrm{~cm}$. from nose.

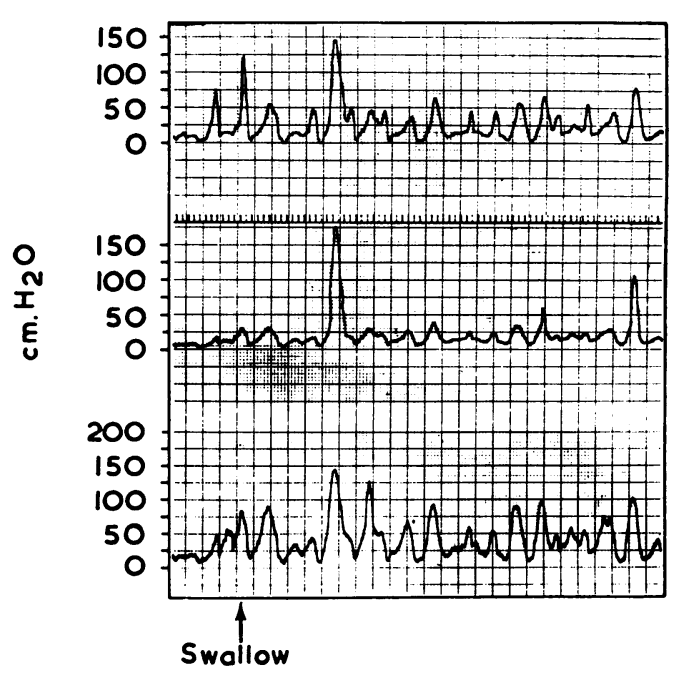

FIG. 9. Grossly abnormal oesophageal pressure tracing from a patient with diffuse oesophageal spasm. Thष peristaltic peaks in all three tracings occur simultaneousl $\overrightarrow{\mathrm{D}}$ and have peak pressures of over $150 \mathrm{~cm}$. of water, which is approximately double the normal value. Upper tracing $28 \mathrm{~cm}$. from nose; middle tracing, $33 \mathrm{~cm}$. from nose lower tracing, $38 \mathrm{~cm}$. from nose. 
OESOPHAGEAL Dilatation In all patients this was the initial method of treatment. In six, oesophagoscopy alone was performed before the diagnosis was established, and in all of these the dilatation produced by simply passing the Negus oesophagoscope resulted in considerable relief of symptoms. In four of these six, the benefit was for only a short time. Where a narrowed segment was encountered it was dilated by bouginage and the Negus hydrostatic dilator. In seven of the 12 patients, oesophagoscopy with dilatation was the only treatment.

LOWER OESOPHAGOMYOTOMY If symptoms were unrelieved by dilatation, or if dilatation was required too frequently, the patient was advised to undergo lower oesophagomyotomy. Five of the 12 patients were treated in this way. The oesophagus was mobilized through a low left lateral thoracotomy, and the muscularis externa was divided with a single longitudinal incision extending from the aortic arch to the proximal stomach.

The findings at operation varied from normal to grossly abnormal. In two of the five patients, the oesophageal muscle was greatly hypertrophied and measured $15 \mathrm{~mm}$. and $11 \mathrm{~mm}$. in thickness respectively. The former was the patient with the large diverticulum whose radiographs are shown in Figure 6. When the diverticulum had been excised a finger inserted into the oesophagus felt as if it was enclosed in a stiff rubber hose. This thickening was greatest in an area beginning $3 \mathrm{~cm}$. above the hiatus oesophagus and extending proximally for $5 \mathrm{~cm}$. The diverticulum was $5 \mathrm{~cm}$. in diameter. In one patient the oesophageal muscle was moderately thickened but in the other two it was macroscopically normal. In each case histological examination showed normal muscle. Autonomic ganglia were seen in two of the specimens.

ASSOCIATED HIATAL HERNIA Of the 12 patients, three had a hiatal hernia, two of the sliding type and one para-oesophageal. The latter had the hernia repaired before it was recognized that she had oesophageal incoordination, which has been subsequently managed by oesophagoscopy and dilatation. Neither of the patients with a sliding hiatal hernia had oesophagitis. Both were subjected to repair of the hernia, and in one a lower oesophagomyotomy was also performed. In the other, no myotomy was carried out in an attempt to see whether dealing with the hernia would improve the dysphagia.

\section{RESULTS}

The assessment of results has been difficult in some cases because these patients learn to live with their disease and often minimize their symptoms. This happens similarly in patients with achalasia, and Ellis (1960) suggested that radiological progress is often a better guide than symptoms when assessing the results of treatment.

With Dilatation ALONE Seven of the 12 patients were managed by dilatation alone and none required hospital admission more than twice a year. The relief of symptoms produced by dilatation usually lasted several months at least and in one it lasted five years. This patient has had five dilatations since 1945, and when last reviewed in December 1965 claimed to be having only occasional dysphagia. She was advised to undergo oesophagomyotomy on two occasions, but declined each time. Four patients had intervals greater than two years between dilatations.

Dilatation made little or no difference to the radiological appearance or to the oesophageal pressure measurements but significantly reduced the severity of symptoms. The patient who had only a hiatal hernia repair had her first oesophageal dilatation in 1951 and, in spite of intermittent minor dysphagia, did not require admission again until 1965, when the hiatal hernia was demonstrated for the first time and subsequently repaired. Her oesophageal incoordination had not shown significant progression in that period.

LOWER OESOPHAGOMYOTOMY Five patients were treated in this way. Myotomy greatly improved the radiological appearances, though it did not completely abolish them. In the oesophageal tracings, the hypertonic, repetitive, non-peristaltic waves were eliminated by myotomy (Fig. 10).

The results in all five patients have been good, with an almost total relief of symptoms and, in two, a weight gain in excess of 2 st. $(12 \cdot 7 \mathrm{~kg}$.). The follow-up periods in these cases have ranged from 12 years to three months. The earliest operation was in 1954 and the latest in 1965. All patients experienced relief of symptoms and only one patient required a further dilatation. This patient, who underwent operation in 1954, recently returned with minor intermittent dysphagia. Radiological examination, oesophageal pressure measurements, and oesophagoscopic findings all suggested that there was significant incoordination above the aortic arch. The oesophagus was dilated and his progress is being watched. 


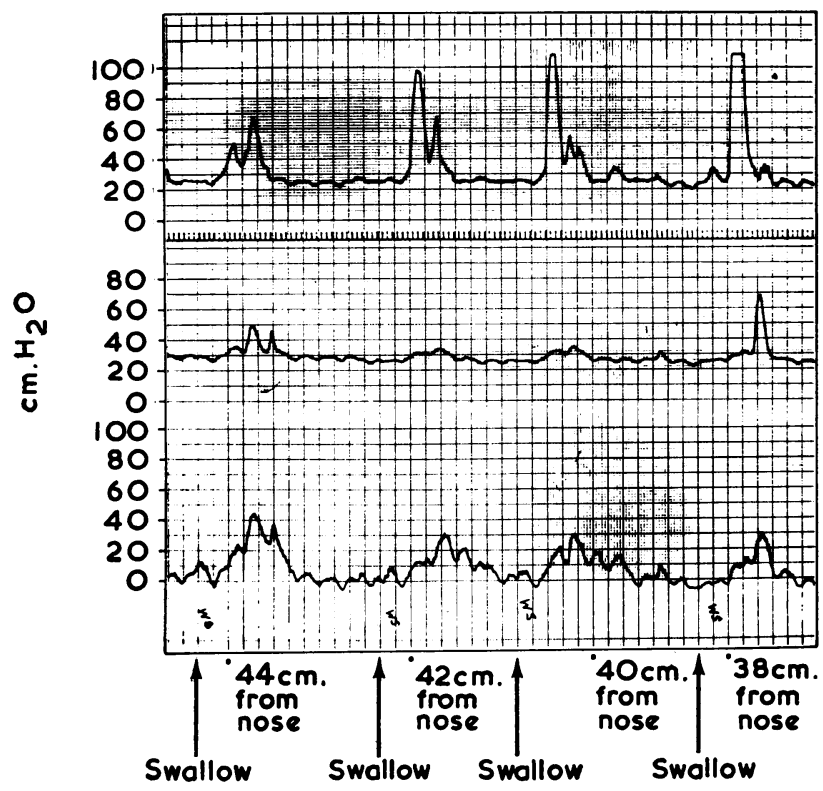

FIG. 10. Oesophageal pressure tracings of $a \stackrel{\text { Fे }}{2}$ patient who had undergone lower oesophago- $\times$ myotomy for diffuse spasm. The abnormal con- tractions have been eliminated below the aortic on arch. Upper tracing, $30 \mathrm{~cm}$. from nose; middle or tracing, $35 \mathrm{~cm}$. from nose; lower tracing, $40 \vec{二}$ $\mathrm{cm}$. from nose.
WITH ASSOCIATED HIATAL HERNIA These three patients are at present free from dysphagia. The patient with the para-oesophageal hernia has been discussed previously. The patient who underwent myotomy and repair of the hernia has now been symptom-free for six months, and the patient who had a hernia repair only has been symptom-free for five months.

\section{DISCUSSION}

Little is known about the aetiology of this condition and all suggested theories lack proof. Many patients without dysphagia show some degree of oesophageal incoordination. However, if the disease progresses to dilatation and diverticulum formation, symptoms probably always occur. When the patient is asymptomatic no treatment is indicated. Once symptoms begin, treatment is necessary. Oesophagoscopic dilatation should be tried initially. If this proves unsatisfactory lower oesophagomyotomy should be undertaken.

Creamer (1962) stated that a sliding hiatal hernia is almost invariably present in cases of diffuse spasm because of the effect of contracting longitudinal muscle which pulls the proximal stomach into the chest. In this series we have not been able to demonstrate such a high incidence of hernia in spite of repeated radiological and endoscopic examinations. A sliding hiatal hernia can cause dysphagia in the absence of ulceration and stricture formation, and where hernia and $\stackrel{\circ}{\varnothing}$ diffuse spasm coexist it may be important to estimate the contribution of each to dysphagia. It was with this idea in mind that one of the two patients with sliding hiatal hernia was subjected to myotomy plus repair of the hernia and the other to a hernia repair alone. As in other forms of oesophageal obstruction there is a tendency for food to become impacted, and three patients suffered from this during the course of the disease.

The relationship of diffuse spasm to achalasia is a close one. Achalasia after dilatation may exhibit a somewhat similar radiological appearance, and it is only by following the course of the disease that the two can ultimately be distinguished.

The normal relaxation of the oesophago-cardiac $N$ sphincter, which is the usual finding in diffuse $N$ spasm, does not occur in achalasia. In one patient No in the series, the first diagnosis was achalasia, but $\stackrel{\omega}{\sigma}$ this was later changed when the characteristic oesophageal pressure tracings and radiological $\stackrel{0}{\overparen{D}}$ appearances of diffuse spasm developed. Non- $\Phi$ peristaltic oesophageal contractions are sometimes found at manometry in people without dysphagia. This is consistent with the finding that some $\vec{\Phi}$ patients demonstrating the corkscrew appearance $\frac{O}{\mathbb{D}}$ radiologically are asymptomatic. However, we have not seen a severe form of this condition 
without symptoms. Early in the series an attempt was made to treat these patients with antispasmodic drugs; but, as most other workers have found, the relief produced was transient. This form of treatment was then abandoned in favour of the two methods discussed above. In this series no patient has died from the disease, illustrating the fact that although this is an unpleasant condition it is not a fatal one.

\section{SUMMARY}

Twelve cases of diffuse oesophageal spasm are presented. Their symptomatology and clinical findings are considered. An attempt has been made to trace the progress of the disease over a period which in some cases exceeds 12 years. Two types of management are outlined and the results are discussed.

\section{REFERENCES}

Creamer, B (1962). In Surgical Physiology of the Gastro-Intestinal Tract: Proc. Symp. roy. Coll. Surg. Elin., p. 47, ed. A.N.Smith. Donoghue, F. E., and Code, C. F. (1958). Pattern of oesophageal motility in diffuse spasm. Gastroenterology, 34, 782.

Ellis, F. G. (1960). The natural history of achalasia of the cardia. Proc. roy. Soc. Med., 53, 663.

Ellis, F. H., Olsen, A. M., Holman, C. B., and Code, C. F. (1958). Surgical treatment of cardiospasm (achalasia of the esophagus): considerations of aspects of esophagomyotomy. J. Amer. med. Ass., 166, 29.

Johnstone, A. S. (1950). In A Text-book of $X$-ray Diagnosis, 2nd ed., ed. S. C. Shanks and P. Kerley, Vol. 3, p. 36. Lewis, London.

Le Roux, B. T., and Wright, J. T. (1961). Cardiospasm. Brit. J. Surg., 48, 619.

Sheinmel, A., Priviteri, C. A., and Poppel, M. H. (1949). A study of the effect of certain drugs on curling of the esophagus: A preliminary report. Amer. J. Roentgenol., 62, 807. 